\title{
Positive Effect of Injectable Platelet Rich Fibrin (i-PRF) on Vital Bone Formation in Graft Reconstruction of the Mandible: A Histologic and Histomorphometric Study
}

${ }^{1}$ Cameron Y. S. Lee, DMD, MD, PHD, MPH, ${ }^{2}$ Hari Prasad, BS, MDT, MS, ${ }^{3}$ Cameron C. Y. Lee, DMD, MD, ${ }^{4}$ Sanjana Prasad, BDS, ${ }^{5}$ Jon B. Suzuki, DDS, PHD, MBA

${ }^{1}$ Private Practice in Oral, Maxillofacial and Reconstructive Surgery. Aiea, Hawaii 96701; Clinical Professor of Surgery, Department of Periodontology/Oral Implantology Kornberg School of Dentistry. Temple University, Philadelphia, PA 19140; ${ }^{2}$ Assistant Research Director and Senior Research Scientist. Hard Tissue Research Laboratory. University of Minnesota School of Dentistry. Minneapolis. MN. 55455; ${ }^{3}$ Oral and Maxillofacial Surgery Resident. Harvard Dental School. Boston, MA 02114; ${ }^{4}$ Assistant Professor. Hard Tissue Research Laboratory. University of Minnesota School of Dentistry. Minneapolis, MN. 55455; ${ }^{5}$ Professor Emeritus. Departments of Periodontology/Oral Implantology and Microbiology/ Immunology. Temple University School of Dentistry and Medicine. Temple University. Philadelphia, PA 19140

\section{ABSTRACT}

In this clinical case series, relative centrifugation force (RCF) and centrifugation time (CT) were reduced to produce the liquid form of platelet rich fibrin (i-PRF). The goal of this study is to histologically evaluate if the liquid form of PRF has a positive effect on vital bone formation in the grafted mandible using mineralized freeze-dried allogeneic bone.

Materials and Methods: Forty-two patients completed immediate bone grafting after a non-restorable endodontically treated molar tooth was extracted in preparation for future implant surgery. Each bone graft was augmented with i-PRF. To process i-PRF, relative centrifugation force (RCF) was reduced from 2,700 RPM to 700 RPM (60g). Centrifugation time was reduced from 12 minutes to 3 minutes. After an average bone graft healing time of 8 to 12 weeks, bone core samples were obtained at the time of implant surgery for histological and histomorphometric analysis.

Results: Forty-two bone cores were harvested at the time of implant surgery for histological and histomorphometric analysis. The liquid form of PRF resulted in greater percentages of vital bone formation (average $87.4 \%$ ) compared to the existing published literature.

Conclusion: i-PRF resulted in greater percentages of new vital bone formation compared to the published literature. This could be due to the continuous slow release and delivery of growth factors in i-PRF. Further clinical use of iPRF is needed to evaluate the full potential of bone regeneration.

Key Words: Injectable Platelet Rich Fibrin (i-PRF). Bone formation. Allogeneic bone. Reduction of Relative Centrifugation Force (RCF). Reduction of Centrifugation Time (CT). Histology. Histomorphometric analysis. 
*Correspondence to Author:

Cameron Y. S. Lee, DMD, MD, PHD, MPH, Private Practice in Oral, Maxillofacial and Reconstructive Surgery. Aiea, Hawaii 96701; Clinical Professor of Surgery, Department of Periodontology/Oral Implantology Kornberg School of Dentistry. Temple University, Philadelphia, PA 19140

\section{How to cite this article:}

Cameron Y. S. Lee, Hari Prasad, Cameron C. Y. Lee, Sanjana Prasad, Jon B. Suzuki. Positive Effect of Injectable Platelet Rich Fibrin (i-PRF) on Vital Bone Formation in Graft Reconstruction of the Mandible: A Histologic and Histomorphometric Study. International Journal of Dental Research and Reviews, 2020, 3:43

\section{Introduction}

No quantitative correlation exists between formation of vital bone and implant success. In any grafting procedure related to successful implant osseointegration, the objective is the formation of $100 \%$ vital bone. ${ }^{1}$ The ideal graft material is osteogenic, osteoconductive and osteoinductive and can be evaluated by histology and histomorphometric methods to calculate percentages of vital bone formation, residual graft material and connective tissue. ${ }^{2,3}$ Bone graft substitute materials currently used include allogeneic, xenogeneic and alloplastic materials. ${ }^{4,5}$ They provide the cellular scaffolding at the surgical site through space maintenance and facilitate the healing response of graft materials. To facilitate bone formation, one strategy is the use of bioactive molecules with bone graft substitute materials. However, this will increase the cost for the patient. To control patient expense, platelet autologous preparations such as platelet rich plasma (PRP) and most recently, platelet rich fibrin (PRF) obtained from the patient's own blood provide an alternative to expensive commercial products. ${ }^{6-8}$

Produced from megakaryocytes in bone marrow, platelets contain lysosomes, dense granules and alpha granules. ${ }^{9-11}$ In the coagulation cascade of hemostasis, platelets terminate bleeding via vascular obliteration and formation of a fibrin clot. ${ }^{12-14}$ With formation of the fibrin clot, the alpha granules release important bioactive molecules such as growth factors, platelet-specific proteins, coagulation factors, adhesion molecules, cytokines, angiogenic factors, proteoglycans and chemokines. ${ }^{15-17}$ Alpha granules contain growth factors such as platelet-derived growth factors $A$ and $B$ (PDGF-AB), transforming growth factor beta (TGF-B), insulin-like growth factor-1 (IGF-1), epidermal growth factor (EGF), and vascular endothelial growth factor (VEGF).6,18,19 For growth factors to have a positive effect on wound healing, high concentrations of growth factors must be obtained.

Bone formation is accelerated by the liberation of specific growth factors contained in the alpha granules of the platelets. In their clinical studies with mandibular reconstruction, Marx et. al. ${ }^{6}$ demonstrated that the addition of PRP resulted in early graft consolidation and mineralization in half the time compared with grafts without the addition of PRP. Bone healing was accelerated approximately 2 times that of autogenous bone grafts without PRP. Bone grafts in general produce a trabecular dense bone that is equal to or greater than that of the nongrafted posterior mandible. Since the first report by Marx and colleagues, other extensive studies on PRP have been published in the literature. ${ }^{18-21}$ However, several studies on PRP reported no wound healing benefit. ${ }^{22-24}$ Inconsistent results in the literature could be due to the many different methods developed in platelet collection and preparation of autologous platelet concentrates that results in variable platelet concentrations. ${ }^{20,25}$ Further, the required use of exogenous thrombin may result in biochemical alteration of PRP and lead to the premature release of growth factors into the wound healing site before osseous healing has been initiated that begins with the presence of osteoblasts. ${ }^{21,} 26$ 
Leucocyte-platelet rich fibrin (L-PRF), is a second-generation autologous platelet concentrate introduced by Choukroun et. al. in 2001 that does not require the use of anticoagulants and exogenous thrombin. ${ }^{26}$ As with platelet-rich plasma, L-PRF is involved in the recruitment and differentiation of cell types such as endothelial cells, osteoblasts, chondrocytes and fibroblsts. ${ }^{27,}{ }^{28} \mathrm{~L}-\mathrm{PRF}$ is prepared from the patient's own venous blood and centrifugation occurs in a glass tube that produces a dense fibrin matrix that not only contains the growth factors and leukocytes, but cytokines such as the interleukins, II-1B, II-4B, II-6B and tumor necrosis factor-alpha that facilitates wound healing through cell proliferation, angiogenesis and extracellular matrix formation. ${ }^{21,26,29-33}$ Release of these biological mediators from the fibrin matrix occurs over a 10-14 day period that is well beyond the first 24 hours observed with PRP. ${ }^{29-31,34,35}$ An additional advantage with L-PRF compared to PRP is that leukocytes have been shown to facilitate osteogenesis, angiogenesis and lymphogenesis. ${ }^{36-38}$

High relative centrifugation force (RCF) of 2,700 rpm and centrifugation time of 12 minutes (700g) is a requisite to produce platelet rich fibrin during the centrifugation process. ${ }^{26,29-31}$ For successful tissue regeneration from L-PRF, a continuous delivery of growth factors to the recipient site must occur. Ghanaati and colleagues ${ }^{39}$ showed that by decreasing the centrifugation speed produced greater numbers of growth factors and leukocytes that are slowly released from the fibrin matrix. This research was followed by the introduction of advanced platelet-rich fibrin (A-PRF) and the liquid form of PRF (i-PRF) that decreased both the centrifugation speed and time. ${ }^{35,40-43}$

In two systematic reviews by Del Fabbro and colleagues ${ }^{44}$ and Dragonas et al. ${ }^{45}$ on ridge augmentation procedures, there is no histomorphometric evidence that autologous platelet concentrates have a positive effect on the dimensions of ridge augmentation/regeneration when mixed with bone graft materials. The paucity of clinical evidence on the effects of L-PRF on new bone formation is greatly needed and is the driving force for this clinical case series. The goal of this study is to histologically evaluate the effects of the liquid form of PRF on vital bone formation in the grafted mandible using mineralized freeze-dried allogeneic bone. To the best of the author's knowledge, there are no published articles in the English language literature evaluating the performance of i-PRF with mineralized freeze-dried allogeneic bone on new vital bone formation in the grafted mandible. The authors hypothesized that i-PRF will have a positive effect on increasing vital bone formation in the grafted mandible compared to other treatments reported in the contemporary published literature that used only allograft with or without autologous concentrates such as PRP, L-PRF and A-PRF.

\section{Materials and Methods}

This case series was completed in a private practice (CYSL) that consisted of 42 patients. Their age range was 42 to 78 years. This study was in accordance with the ethical standards of the institutional and national research committee and with the Helsinki Declaration of 1975 that was revised in $2000 .{ }^{46}$ No ethical approval was required as this is a case series and the identity of all human participants were not identified.

Inclusion Criteria: Patients included in this study consisted of the following criteria: a failed endodontically treated first or second mandibular molar tooth that was determined to be fractured and unrestorable.The gingival tissues were edematous and erythematous with fistula formation. The mandible demonstrated clinical signs of infection with greater than $50 \%$ loss of the buccal cortical plate compared to the vertical depth of the extraction socket after debridement of the intraosseous infection. Purulent discharge from the fistula was not an absolute clinical finding. Radiographic criteria using both panoramic radiography and cone beam computed tomography included a diffuse radiolucency around the roots of the molar teeth consistent with an intraosseous infection.

Exclusion Criteria: Patients who were pregnant. Patients in psychiatric treatment. Patients with a 
past or current history of treatment with bisphosphonates, chemotherapy and RANKL inhibitors were also excluded from the study.

\section{Preparation of i-PRF and Bone Graft Material}

Venous blood was obtained from the antecubital fossa of the upper extremity from each patient enrolled in the study. Blood was collected in two $10 \mathrm{ml}$ sterile glass tubes without the addition of any anticoagulant and immediately placed in the centrifuge for processing of liquid L-PRF. Centrifugation parameters were set at $700 \mathrm{rpm}$ for 3 minutes that resulted in approximately $1 \mathrm{ml}$ of the liquid form of L-PRF at the most superior layer. As i-PRF is produced as a liquid, it was aspirated from the glass tubes using a 25-gauge sterile needle attached to a $3 \mathrm{ml}$ plastic syringe. The liquid form of L-PRF is to be sprayed on to the graft material before the graft material is mixed and placed in the bone graft recipient site.

\section{Surgical Operative Technique}

All patients rinsed with chlorhexidine gluconate $0.12 \%$ prior to the surgical procedure for 45 seconds. Under local anesthesia, surgical extraction of endodontically treated first and second mandibular molars was completed by only one oral and maxillofacial surgeon (CYSL). With completion of the extraction of the molar tooth and preparation of the bone graft recipient site, $0.5 \mathrm{cc}$ to $1.0 \mathrm{cc}$ of mineralized cortical freeze dried allogeneic bone (LifeNet Health, Virginia Beach, VA) was loosely packed into the molar recipient site up to the alveolar crest after the i-PRF was mixed with the graft material. The graft material was covered with a titanium-reinforced high- density polytetrafluoroethylene (dPTFE) membrane (Cytoplast Ti-250 Titanium-Reinforce Dense Membrane, Osteogenics Biomedical, Lubbuck, TX).

\section{Histologic Processing}

For each patient, bone graft healing occurred over an 8 to 12-week period to allow bone remodeling in preparation for implant surgery. A trephine drill with a $2 \mathrm{~mm}$ internal diameter and length of $10 \mathrm{~mm}$ was used to harvest all bone cores of the mandible. Each core bone biopsy contained only the grafted area and no native bone from the mandible. Biopsy samples were fixed in $10 \%$ buffered formalin and submitted for histologic examination. Upon receipt in the Hard Tissue Research Laboratory of the University of Minnesota, all specimens were immediately dehydrated with a graded series of alcohols for 9 days. After dehydration, the specimens were infiltrated with a light-curing embedding resin (Technovit 7200 VLC, Kulzer, Wehrheim, Germany). After 20 days of infiltration with constant shaking at normal atmospheric pressure, the specimens were embedded and polymerized by $450 \mathrm{~nm}$ light with the temperature of the specimens never exceeding $40^{\circ} \mathrm{C}$. The specimens were then prepared by the cutting/grinding method of Rohrer and Schubert. ${ }^{47}$ The specimens were cut to a thickness of $150 \mu \mathrm{m}$ on an EXAKT cutting/grinding system (EXAKT Technologies, OK). Slides were polished to a thickness of $45 \mu \mathrm{m}$ with a series of polishing sandpaper discs from 800 to 2400 grit using the EXAKT microgrinding system followed by $3 \mu \mathrm{m}$ alumina polishing paste. The slides were stained with Stevenel's blue and Van Gieson's picro fuchsin for histologic analysis.

Microphotographs were obtained, scanned, digitized and analyzed using a NIKON ECLIPSE 50i microscope (Nikon Corp., Japan) and a SPOT INSIGHT 2 mega sample digital camera (Diagnostic Instruments, Inc., USA). Histomorphometric measurements were completed using a combination of programs SPOT INSIGHT 2 mega sample digital camera, (Adobe Photoshop, Adobe Systems, USA) and a public domain image program (NIH Image, National Institutes of Health, USA). The following parameters were measured in terms of the percentage of the primary area of interest: total primary area, new bone formation, residual graft material (mineralized cortical allograft), and bone marrow. The presence of osteocytes in lacunae was identified as vital bone formation compared to 
residual graft material that did not contain osteocytes. Vital bone and mineralized allograft were combined as the total bone area.

\section{Histologic Analysis and Histomorphometry}

The primary variable evaluated is vital bone formation reported as the percentage of newly formed bone assessed from histology and histomorphometry. Secondary variables analyzed were percent residual graft material and percent bone marrow.

\section{Statistical Analysis}

Statistical analysis reported averages and ranges of percent total bone volume, percent new vital bone formation, percent residual graft material and percent bone marrow formation (Table 1).

\section{Results}

Forty-two patients (10 male and 32 female) were enrolled in this clinical case series. The mean age was 63 years old. Bone cores were obtained 8 to 12 weeks, post-surgery. All grafts and implants healed without complication.

\section{Histologic and Histomorphometric Findings}

Representative images of de novo bone formation are illustrated in Figures 1 to 5 . Histomorphometric analysis (Table 1) of the 42 allogeneic bone cores revealed a $49.3 \%$ average total bone volume, of which an average of $87.4 \%$ was new vital bone (range $23 \%$ to $100 \%$ ). Further analysis revealed $12.6 \%$ residual graft particles and $50.7 \%$ bone marrow. Direct contact of allogeneic and newly formed autogenous bone was often observed due to bridging of allograft particles (AG) and vital new bone (NB) distinguished by osteocytes (OC) in the lacunae (Figure 1).

\section{DISCUSSION}

The available published literature has shown that using the standard protocol to produce L-PRF improves alveolar bone volume on maxillary sinus grafting and alveolar bone augmentation/regeneration in preparation for dental implant placement. Using mineralized freeze-dried allograft with LPRF, Choukroun et al reported $31.2 \%$ new bone formation after four months of sinus graft healing. ${ }^{48}$ Mazor et al reported $33.5 \%$ new bone formation after 6 months of graft healing with L-PRF as the sole graft material. ${ }^{49}$

Choukroun and Ghanaati using a novel low-speed centrifugation concept reported an increase in the number of platelets, growth factors and leukocytes within the fibrin matrix clot. ${ }^{40}$ Altering the centrifugation process by decreasing RCF while keeping centrifugation time constant enriches the platelet, growth factor and leukocyte population. Clark et al. reported that reducing the centrifugation force down to 1,300 RPM and centrifugation time to 8 minutes from the standard protocol results in lower g-forces that allows for greater amounts of growth factor and leukocyte release from the L-PRF fibrin matrix. ${ }^{50}$ Modifying the L-PRF preparation protocol resulted in significantly greater amounts of vital bone formation (46\%) compared to three other modifications of centrifugation force and time in their ridge preservation study to replace non-molar teeth with or without the use of allogeneic bone.

The results of this case series demonstrate an average of $87.4 \%$ new vital bone formation 8 to 12 -weeks post-surgery with i-PRF. Mineralized cortical lamellar bone is observed as early as 8 weeks surrounded by new woven immature bone (Figure 2). Note the presence of green staining osteoid (OD) and the numerous osteocytes (OC) trapped within the osteoid. Numerous osteoblasts (OB) are lined up along the osteoid (OD), all involved in osteogenesis (Figure 3). Neovascularization is also occurring in the connective tissue as illustrated by the formation of blood vessels (BV).

Distinguishing de novo autogenous bone from residual allograft particles can be difficult as both are mineralized. However, bone polarization patterns can differentiate mature lamellar bone from 
immature woven bone (Figure 4) as osteoblastic rimming and osteoid are present in areas of new bone formation as early as eight weeks post-grafting. Such histology can differentiate new bone formation from residual allograft particles demonstrating the osteogenic effect of iPRF on vital bone formation.

Compared to the published literature, the formation of very high percentages of new vital bone formation during the 8 to 12-week healing time may be attributed to the enhanced and prolonged healing effects of the liquid form of PRF. It is theorized that lower $\mathrm{g}$-forces lead to the continuous release and delivery of growth factors at the bone graft recipient site that facilitates angiogenesis (Figure 3), stem cell release, osteoblast differentiation (Figure 5) and proliferation that leads to increased bone formation. ${ }^{21,35,40-42,51-53}$

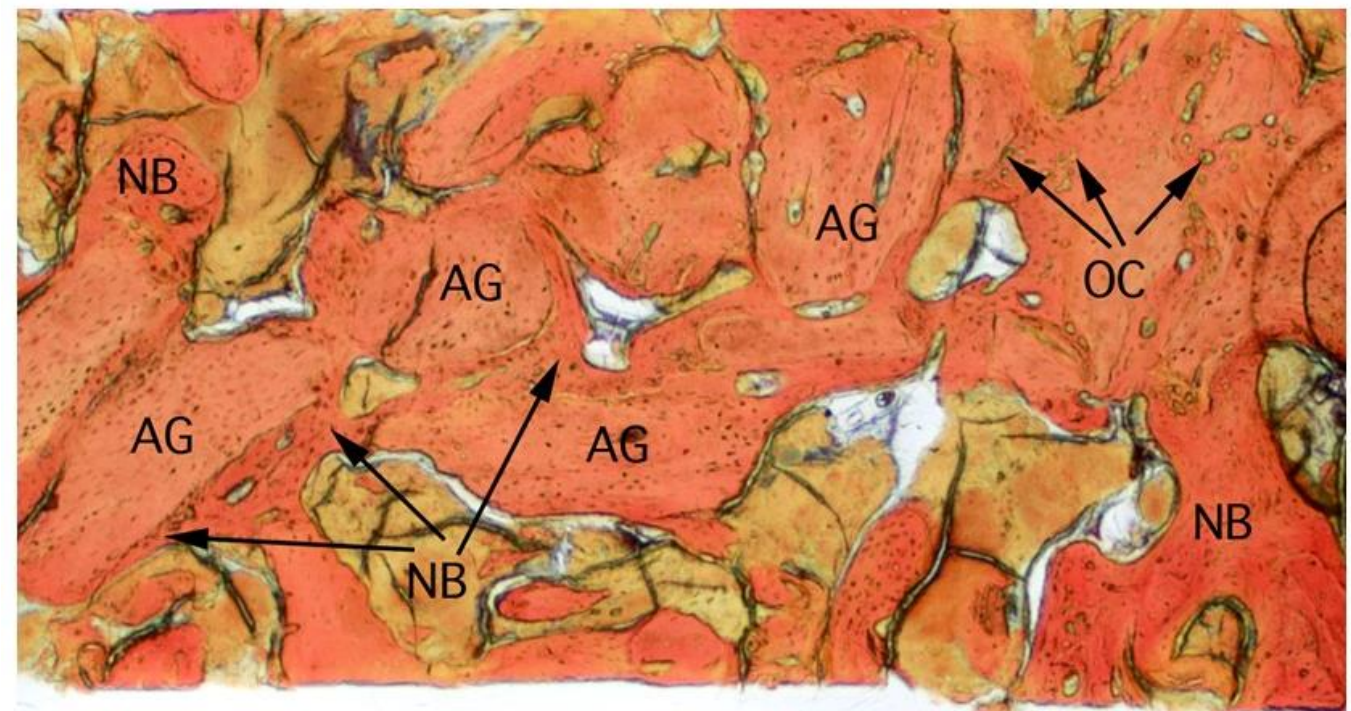

Figure 1. Medium power view shows new bone (NB) formation surrounding particles of allograft material (AG). Note bridging between allograft particles (AG). (x 40, Stevenel's blue and Van Gieson's picro fuchsin)

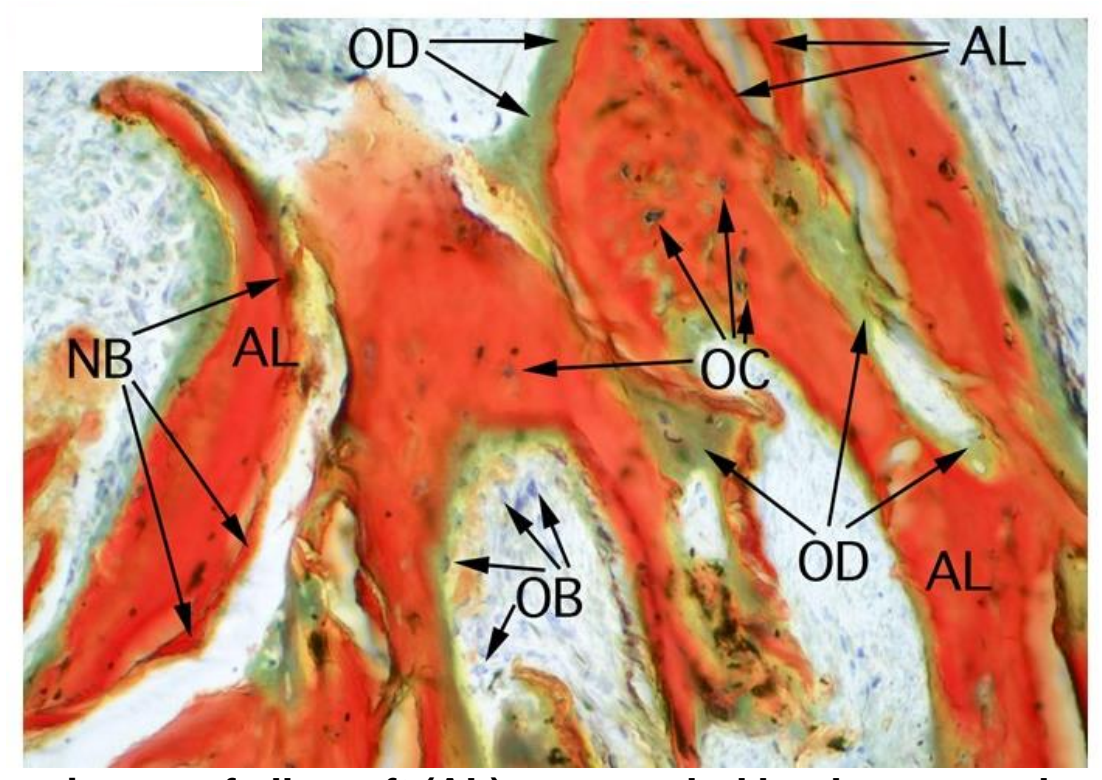

Figure 2. High power image of allograft (AL) surrounded by dense new bone (NB) with a wide seam of green-staining osteoid (OD) lined by osteoblasts (OB). Numerous osteocytes (OC) in their lacunae are irregularly arranged in immature woven bone (NB). (x200, Stevenel's blue and Van Gieson's picro fuchsin) 
Cameron Y. S. Lee et al., IJDRR, 2020 3:43

Table 1 Patient Histomorphometric Results ( $\mathrm{n}=42$ )

\begin{tabular}{|c|c|c|}
\hline Vital Bone (\%) & Residual Graft Material (\%) & Bone Marrow (\%) \\
\hline 97 & 3 & 45 \\
\hline 87 & 13 & 62 \\
\hline 58 & 42 & 55 \\
\hline 97 & 3 & 62 \\
\hline 100 & 0 & 62 \\
\hline 100 & 0 & 55 \\
\hline 85 & 15 & 49 \\
\hline 95 & 5 & 48 \\
\hline 100 & 0 & 54 \\
\hline 75 & 25 & 35 \\
\hline 100 & 0 & 52 \\
\hline 65 & 35 & 23 \\
\hline 100 & 0 & 46 \\
\hline 74 & 26 & 49 \\
\hline 100 & 0 & 50 \\
\hline 93 & 7 & 53 \\
\hline 98 & 2 & 65 \\
\hline 94 & 6 & 62 \\
\hline 93 & 7 & 28 \\
\hline 47 & 53 & 41 \\
\hline 95 & 5 & 40 \\
\hline 95 & 5 & 63 \\
\hline 97 & 3 & 59 \\
\hline 95 & 5 & 58 \\
\hline 84 & 16 & 50 \\
\hline 87 & 13 & 65 \\
\hline 53 & 47 & 60 \\
\hline 99 & 1 & 53 \\
\hline 65 & 35 & 55 \\
\hline 100 & 0 & 41 \\
\hline 100 & 0 & 47 \\
\hline 100 & 0 & 41 \\
\hline 83 & 17 & 55 \\
\hline 99 & 1 & 50 \\
\hline 87 & 13 & 37 \\
\hline 98 & 2 & 31 \\
\hline 100 & 0 & 63 \\
\hline 100 & 0 & 69 \\
\hline 100 & 0 & 27 \\
\hline 100 & 0 & 56 \\
\hline 23 & 77 & 44 \\
\hline 53 & 47 & 70 \\
\hline
\end{tabular}

Table 2. Histomorphometric Averages (\%) $(n=42)$

\begin{tabular}{|l|l|l|}
\hline Vital Bone & Residual Graft Material & Bone Marrow \\
\hline$(23-100) 87.4$ & $(0-77) 12.6$ & $(23-70) 50.7$ \\
\hline
\end{tabular}




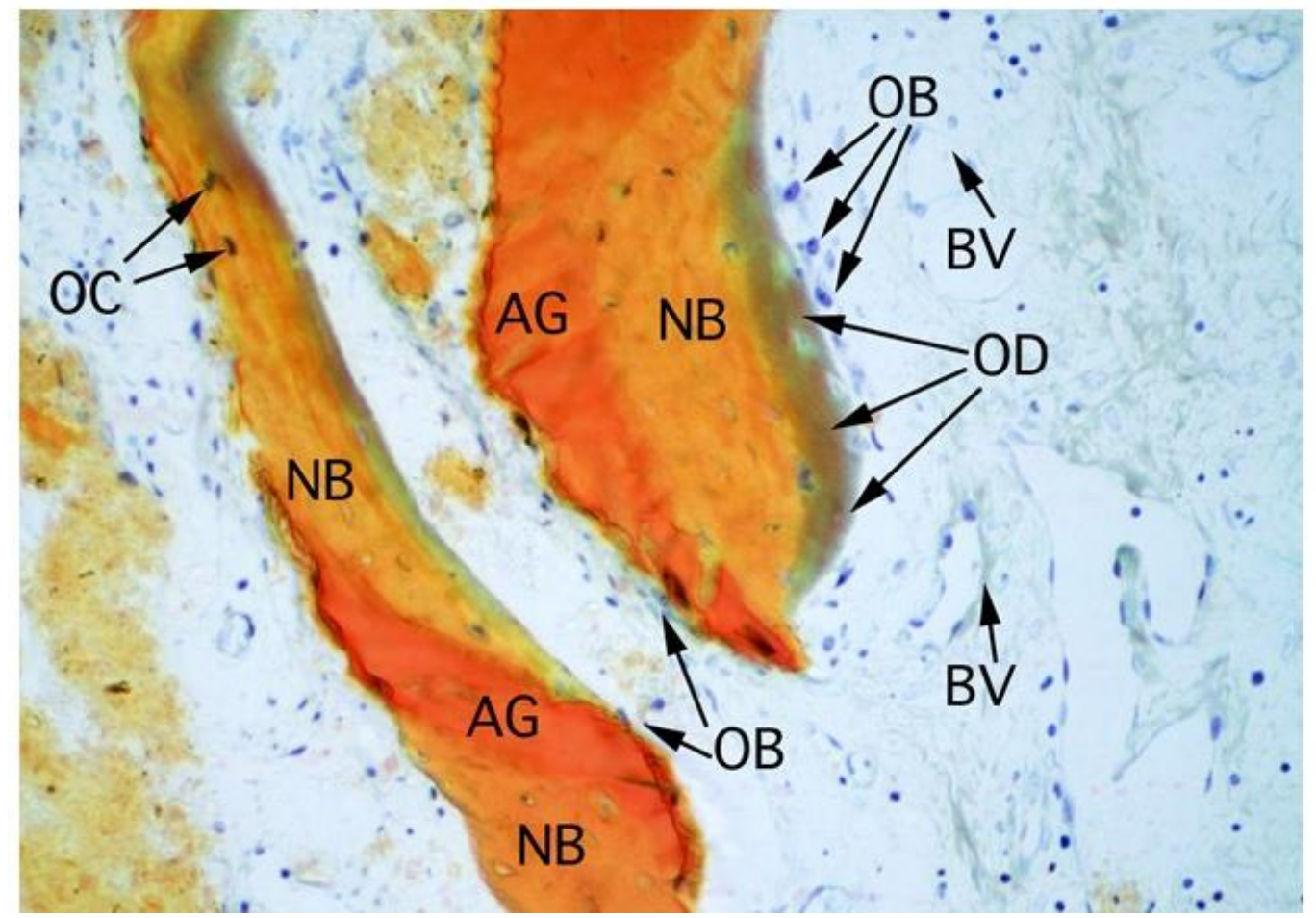

Figure 3. High power image reveals several processes occurring simultaneously. Osteogenesis illustrated by the presence of green staining osteoid (OD) and the numerous osteocytes $(\mathrm{OC})$ trapped within the osteoid. Note the numerous osteoblasts (OB) lined up along the osteoid (OD) all involved in the formation of new bone (NB). Neovascularization is also occurring in the connective tissue as illustrated by the formation of blood vessels (BV). (x200, Stevenel's blue and Van Gieson's picro fuchsin)

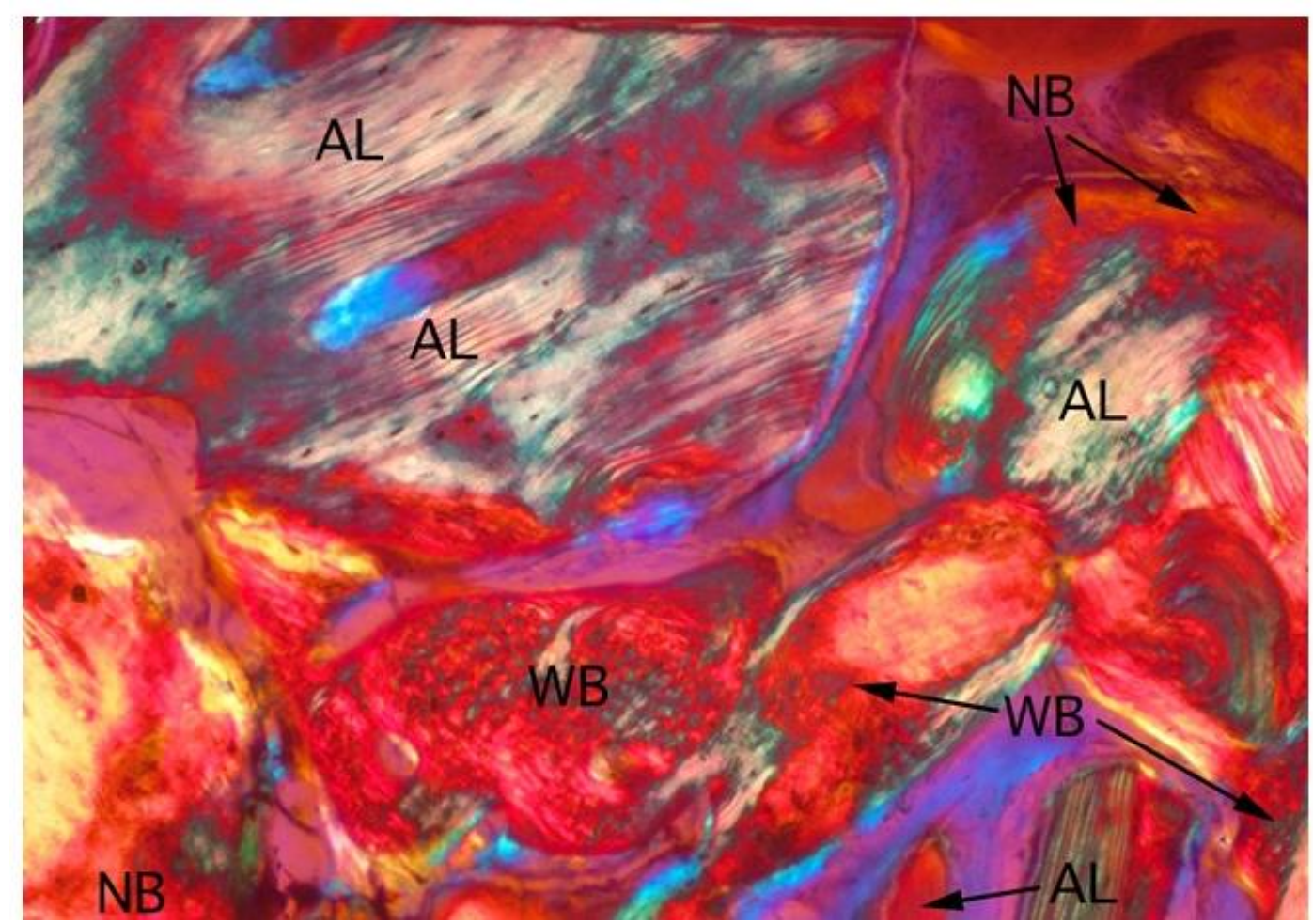

Figure 4. High power polarized image differentiating mature new bone (NB), remaining allograft particles (AG) and immature woven bone (WB). (x100, Stevenel's blue and Van Gieson's picro fuchsin) 


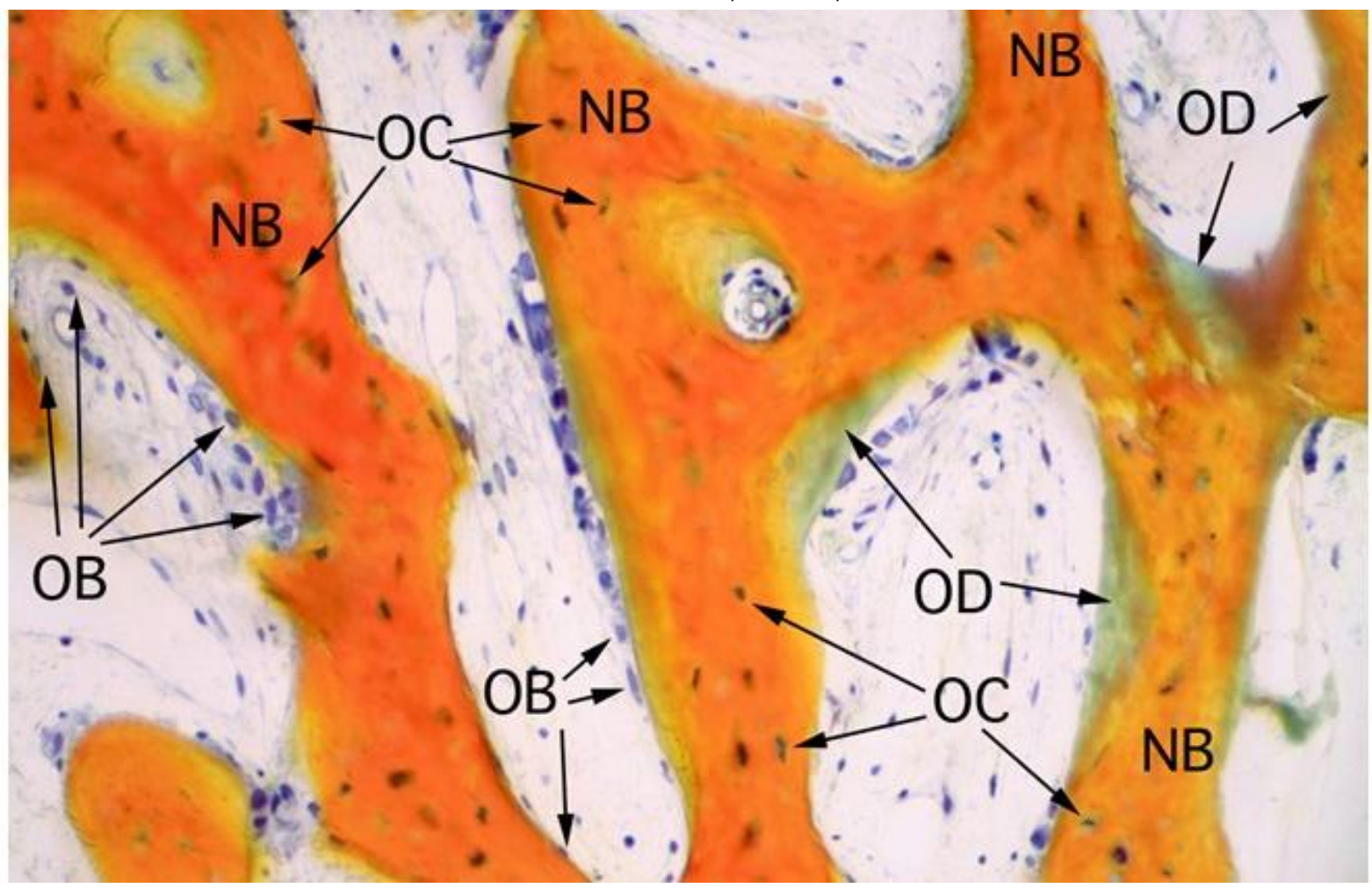

Figure 5. High power image shows osteoblasts (OB) lining osteoid (OD) in areas of new bone (NB) formation. Note the numerous osteocytes (OC) in their lacunae irregularly arranged in immature bone. (x200, Stevenel's blue and Van Gieson's picro fuchsin)

To the best of our knowledge, this is the first study that demonstrates the enhanced and positive effects of the liquid form of PRF on new vital bone formation. Such high percentages of new vital bone formation may allow immediate loading due to primary implant stability based on resonance frequency analysis. This is a study that is currently in progress to determine if there is a definite advantage to surgical placement of dental implants in high percentages of newly formed vital bone and immediate loading of the definitive restoration.

This study does have limitations. The total number of patients enrolled in this study is small, but comparable to other studies. We did not have a control group that could compare bone graft regeneration with and without i-PRF as this is a case series that compared the results to the published literature. Further, it is difficult to compare results of bone regeneration of the maxillary sinus and posterior mandible due to anatomic differences. Such anatomic differences of bone density and vascularization patterns may have different effects on autologous concentrates and substitute graft materials in tissue engineering as demonstrated by histomorphometry in the published literature. Lastly, it is difficult to compare the formation of new vital bone using different graft materials with or without autologous concentrates.

\section{Conclusion}

Based on the results of this clinical case series, i-PRF did result in high percentages of new vital bone as demonstrated histologically and histomorphologically. Further research using randomized cohort studies are needed to fully validate the full osteogenic effects of i-PRF on vital bone formation using different graft materials.

\section{Disclosure}

The authors report no financial interest in any company or products mentioned in this article. 


\section{References}

1. Garetto LP, Chen J, Parr JA, et al. Remodeling dynamics of bone supporting rigidly fixed titanium implants: A histomorphometric comparison in four species including humans. Implant Dent. 1995;4: 235-243.

2. Block MS, Kent JN. Sinus augmentation for dental implants: The use of autogenous bone. J Oral Maxillofac Surg. 1997; 55:1281-1286.

3. Del Fabbro M, Testori T, Francetti $L$, et al. Systematic review of survival rates for implants placed in the grafted maxillary sinus. Int J Periodontics Restorative Dent. 2004; 24:565-578.

4. Susin C, Wikesjo UM. Regenerative periodontal therapy: 30 years of lessons learned and unlearned. Periodontol 2000. 2013; 62: 232-242.

5. Avila-Ortiz G, Elangovan S, Kramer KW, et al. Effect of alveolar ridge preservation after tooth extraction: a systematic review and meta-analysis. J Dent Res. 2014; 93: 950-958.

6. Marx RE, Carlson ER, Eichstaedt RM, et al. Platelet-rich plasma: Growth factor enhancement for bone grafts. Oral Surg Oral Med Oral Pathol Oral Radiol Endod. 1998; 85: 638-646.

7. Bhanot S, Alex JC. Current applications of platelet gels in facial plastic surgery. Facial Plast Surg. 2002; 18: 2733.

8. Eppley BL, Pietrzak WS, Blanton M. Platelet-rich plasma: a review of biology and application in plastic surgery. Past Reconstr Surg. 2006; 118: 147e-159e.

9. Oryan A, Alidadi S, Moshir A. Current concerns regarding healing of bone defects. Hard Tissue. 2013; 2:13.

10. Oryan A, Alidadi S, Moshiri A, et al. Bone regenerative medicine: class options, novel strategies and future directions. J Orthop Surg Res. 2014; 9:18.

11. Bigham-Sadegh A, Oryan A. Basic concepts regarding fracture healing and the current options and future directions in managing bone fractures. Int Wound J. 2015; 12: 238-247.

12. Gosain A, DiPietro LA. Aging and wound healing. World J Surg. 2004; 28: 321-326.

13. Eming SA, Brachvogel B, Odorisio T, et al. Regulation of angiogenesis: wound healing as a model. Prog Histochem Cytochem. 2007; 42: 115-170.

14. Guo S, DiPietro LA. Factors affecting wound healing. J Dent Res. 2010; 89: 219-229.

15. Nurden A. Platelets, inflammation and tissue regeneration. Thromb Haemost. 2011; 105: S13-S33.

16. Ogundipe OK, Ugboko VI, Owotade FJ. Can autologous platelet-rich plasma gel enhance healing after surgical extraction of mandibular third molars. J Oral Maxillofac Surg. 2011; 69: 2305-2310.

17. Kiran NK, Mukunda KS, Tilak Raj TN. Platelet concentrates: a promising innovation in dentistry. J Dent Sci Res. 2011; 2: 50-61.

18. Landesberg R, Roy M, Glickman RS. Quantification of growth factor levels using a simplified method of plateletrich plasma gel preparation. J Oral Maxillofac Surg. 2000; 58: 297-300.

19. Aghaloo TL, Moy PK, Freymiller EG. Investigation of platelet-rich plasma in rabbit cranial defects: A pilot study. J Oral Maxillofac Surg, 2002; 60: 1176-1181.

20. Freymiller EG, Aghaloo TL. Platelet-rich plasma: Ready or not? J Oral Maxillofac Surg. 2004; 62: 484-488.

21. He L, Lin Y, Hu X, et al. A comparative study of platelet-rich fibrin (PRF) and platelet-rich plasma (PRP) on the effect of proliferation and differentiation of rat osteoblasts in vitro. Oral Surg Oral Med Oral Pathol Oral Radiol Endod. 2009; 108: 707-713.

22. Gurbuzer B, Pikdoken L, Tunah M, et al. Scintigraphic evaluation of osteoblastic activity in extraction sockets treated with platelet-rich fibrin. J Oral Maxillofac Surg. 2010; 68:980.

23. Celio-Mariano R, deMelo WM, Carneiro-Avelino C. Comparative radiographic evaluation of alveolar bone healing associated with autologous platelet-rich plasma after impacted third molar surgery. J Oral Maxillofac Surg. 2012; 70: 19-.

24. Choi BK, Im CJ, Huh JY, et al. Effect of platelet-rich plasma on bone regeneration in autogenous bone graft. Int $\mathrm{J}$ Oral Maxillofac Surg. 2004; 33:56-59.

25. Del Corso M, Vervelle A, Simonpieri $A$, et al. Current knowledge and perspectives for the use of platelet-rich plasma (PRP and platelet-rich fibrin (PRF) in oral and maxillofacial surgery part 1: Periodontal and dentoalveolar surgery. Curr Pharm Biotechnol. 2012; 13: 1207-1230.

IJDRR: https://escipub.com/international-journal-of-dental-research-and-reviews/ 
Cameron Y. S. Lee et al., IJDRR, 2020 3:43

26. Choukroun J, Diss A, Simonpieri A, et al. Platelet-rich fibrin (PRF): a second-generation platelet concentrate. Part V: Histologic evaluations of PRF effects on bone allograft maturation in sinus lift. Oral Surg Oral Med Oral Pathol Oral Radiol Endod. 2006a; 101: 299-303.

27. Roy S, Driggs J, Elgharably $\mathrm{H}$, et al. Platelet-rich fibrin matrix improves wound angiogenesis via inducing endothelial cell proliferation. Wound Repair Regen. 2011; 19: 753-766.

28. Chen FM, Wu LA, Zhang M, et al. Homing of endogenous stem/progenitor cells for in situ tissue regeneration: Promises, strategies, and translational perspectives. Biomaterials. 2011; 32: 3189-3209.

29. Dohan DM, Choukroun J, Diss A, et al. Platelet-rich fibrin (PRF): a second-generation platelet concentrate. Part I: technological concepts and evolution. Oral Surg Oral Med Oral Pathol Oral Radiol Endod. 2006a; 101: e37e44.

30. Dohan DM, Choukroun J, Diss A, et al. Platelet-rich fibrin (PRF): A second generation platelet concentrate. Part II: Platelet-related biologic features. Oral Surg Oral Med Oral Pathol Oral Radiol Endod. 2006b; 101: e45-50.

31. Dohan Ehrenfest DM, Del Corso M. Diss A, et al. Three-dimensional architecture and cell composition of a Choukroun's platelet rich fibrin clot and membrane. J Periodontol. 2010; 81: 546-555.

32. Pradeep AR, Rao NS, Agarwal E, et al. Comparative evaluation of autologous platelet-rich fibrin and plateletrich plasma in the treatment of 3-wall intrabony defects in chronic periodontitis: A randomized controlled clinical trial. J Periodontol. 2012; 83: 1499-1507.

33. Bajaj P, Pradeep AR, Agarwal E, et al. Comparative evaluation of autologous platelet-rich fibrin and plateletrich plasma in the treatment of mandibular degree II furcation defects: A randomized controlled clinical trial. J Periodontol Res. 2013; 48:573-581.

34. Kobayashi E, Fluckiger L, Fujioka-Kobayashi, et al. Comparative release of growth factors from PRP, PRF and advanced-PRF. Clin Oral Investig. 2016; 20: 2353-2360.

35. Fujioka-Kobayashi M, Miron RJ, Hernandez M, et al. Optimized platelet-rich fibrin with the low-speed concept: Growth factor release, biocompatibility, and cellular response. J Periodontol. 2017; 88: 112-121.

36. Dohan DM, Choukroun J, Diss, et al. Platelet-rich fibrin (PRF): a second-generation platelet concentrate. Part III: leucocyte activation: a new feature for platelet concentrates? Oral Surg Oral Med Oral Pathol Oral Radiol Endod. 2006c; 101: e51-55.

37. Kawazoe T, Kim HH. Tissue augmentation by white blood cell-containing platelet-rich plasma. Cell Transplant. 2012; 21: 601-607.

38. Soloviev DA, Hazen SL, Szpak D, et al. Dual role of the leukocyte integrin M2 in angiogenesis. J Immunol. 2014; 193: 4712-4721.

39. Ghanaati S, Booms P, Orlowska A, et al. Advanced platelet-rich fibrin: A new concept for cell-based tissue engineering by means of inflammatory cells. J Oral Implantol. 2014; 40: 679-689.

40. Choukroun J, Ghanaati S. Reduction of relative centrifugation force within injectable platelet-rich fibrin (PRF) concentrates advances patients own inflammatory cells, platelets, and growth factors: the first introduction to the low speed centrifugation concept. Eur J Trauma Emerg Surg. 2018; 44: 87-95.

41. Ghanaati S, Al-Maawi S, Herra-Vizcaino C, et al. A proof of the low speed centrifugation speed concept in rodents: New perspectives for in vivo research. Tissue Engineering. Part C, Methods. 2018; 24: 659-670.

42. Elbagdadi $\mathrm{K}$, Kubesch $\mathrm{A}, \mathrm{Yu} \mathrm{X}$, et al. Reduction of relative centrifugal forces increases growth factor release within solid platelet-rich fibrin (PRF)-based matrices: A proof of concept of LSCC (low speed centrifugation concept). Euro J Trauma and Emer Surg. 2017; ttps//doi.org/10.1007/s00068-017-0785-7.

43. Kubesch A, Barbeck M, Al-Maawi S, et al. A low-speed centrifugation concept leads to cell accumulation and vascularization of solid platelet-rich fibrin: An experimental study in vivo. Platelets. 2018; 30: 329-340.

44. Del Fabbro M, Bortolin M, Taschieri S. I autologous platelet concentrate beneficial got post-extraction healing> A systematic review. Int J Oral Maxillofac Surg. 2011; 40: 891-900.

45. Dragonas $\mathrm{P}$, Katsaros $\mathrm{T}$, Avila-Ortiz $\mathrm{L}$, et al. Effects of leukocyte-platelet rich fibrin (L-PRF) in different intraoral bone grafting procedures: a systematic review. Int J Oral Maxillofac Surg. 2019; 48: 250-292.

46. World Medical Association Declaration of Helsinki Ethical Principles for Medical Research Involving Human Subjects. World Medical Association Declaration of Helsinki: Ethical principles for medical research involving human subjects. JAMA. 2000; 284: 3043-3045. 
Cameron Y. S. Lee et al., IJDRR, 2020 3:43

47. Rohrer MD, Schubert CC. The cutting-grinding technique for histological preparation of undecalcified bone and bone-anchored implants: Improvement in instrumentation and procedures. Oral Surg Oral Med Oral Pathol, 1992; 74: 73-78.

48. Choukroun J, Diss A, Simonpierri A, etal. Platelet-richfibrin(PRF): Asecond- generation platelet concentrate. Part V: Histologic evaluations of PRF effects on bone allograft maturation in sinus lift. Oral Surg Oral Med Oral Pathol Oral Radiol Endod. 2006b; 101:299-303.

49. Mazor Z, Horowitz RA, Del Corso M, et al. Sinus floor augmentation with simultaneous implant placement using Choukroun's platelet-rich fibrin as the sole grafting material: a radiologic and histologic study at 6 months. J Periodontol. 2009; 80: 2056-2064.

50. Clark D, Rajendran Y, Paydar S, et al. Advanced platelet-rich fibrin and freeze-dried bone allograft for ridge preservation: A randomized controlled clinical trial. J Periodontol. 2018; 89: 379-387.

51. Kanno T, Takahashi T, Tsujisawa T, et al. Platelet-rich plasma enhances human osteoblast-like cell proliferation and differentiation. J Oral Maxillofac Surg, 2005; 63: 362-369.

52. Miron RJ, Fujioka-Kobayashi M, Hernandez M, et al. Injectable platelet-rich fibrin (i-PRF): Opportunities in regenerative dentistry? Clin Oral Investig. 2017; 21: 2619-2627.

53. Miron RJ, Fujioka-Kobayashi M, Bishra M, et al. Platelet-rich fibrin and soft tissue wound healing: A systematic review. Tissue Eng Part B Rev. 2017; 23:83-99. 